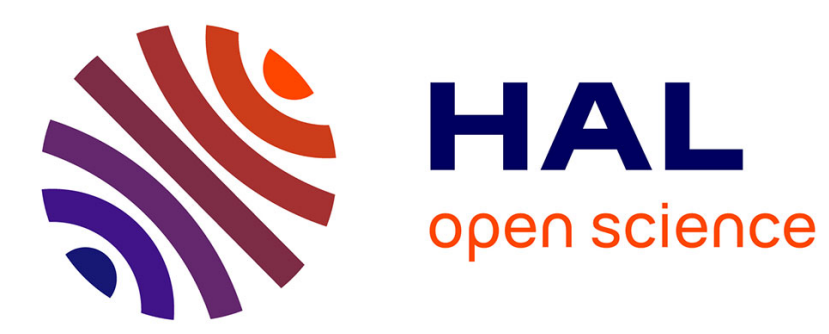

\title{
Artère stapédienne persistante dans le cadre d'un syndrome PHACE
}

R. Quatre, P. Manipoud, S. Schmerber

\section{To cite this version:}

R. Quatre, P. Manipoud, S. Schmerber. Artère stapédienne persistante dans le cadre d'un syndrome PHACE. Annales françaises d'Oto-rhino-laryngologie et de Pathologie Cervico-faciale, 2019, 136, pp.212 - 214. 10.1016/j.aforl.2018.07.004 . hal-03484413

\section{HAL Id: hal-03484413 \\ https://hal.science/hal-03484413}

Submitted on 20 Dec 2021

HAL is a multi-disciplinary open access archive for the deposit and dissemination of scientific research documents, whether they are published or not. The documents may come from teaching and research institutions in France or abroad, or from public or private research centers.
L'archive ouverte pluridisciplinaire HAL, est destinée au dépôt et à la diffusion de documents scientifiques de niveau recherche, publiés ou non, émanant des établissements d'enseignement et de recherche français ou étrangers, des laboratoires publics ou privés.

\section{다)(1) $(5$}

Distributed under a Creative Commons Attribution - NonCommercial| 4.0 International 


\title{
Artère stapédienne persistante dans le cadre d'un syndrome PHACE
}

\author{
R. Quatre ${ }^{1,2,3^{*}}$, P. Manipoud ${ }^{3}$, S. Schmerber ${ }^{1,2,4}$
}

1: Clinique universitaire d'oto-rhino-laryngologie et de chirurgie cervico-faciale, pôle PALCROS, CHU de Grenoble, hôpital Nord, CS 10217, Grenoble cedex 9,

France

2: Pôle médecine, Université Grenoble Alpes, domaine de la Merci, 38700 La Tronche, France

3: Service d'oto-rhino-laryngologie et de chirurgie cervico-faciale, Centre Hospitalier Métropole Savoie, 73000 Chambéry

4: Université Grenoble-Alpes, Brain Tech Lab, Inserm UMR 1205, 38000 Grenoble, France

* Auteur Correspondant

Email: rquatre@ chu-grenoble.fr (Raphaële QUATRE)

Département d'oto-rhino-laryngologie et de chirurgie cervico-faciale, pôle PALCROS, CHU de Grenoble, hôpital Nord, CS 10217, Grenoble cedex 9, France.

Tel: +33614531563, Fax: +33476765120 


\section{Résumé}

Introduction : le syndrome PHACE est un syndrome rare caractérisé par des anomalies de la fosse postérieure, des hémangiomes, des anomalies artérielles, une coarctation de l'aorte et des anomalies oculaires.

Cas clinique : nous rapportons le cas d'une enfant de 6 ans suivie depuis son enfance pour un syndrome PHACE avec hémangiomes hémifaciaux gauche. Elle s'est présentée en consultation ORL pour une hypoacousie gauche. La tomodensitométrie a permis de mettre en évidence une artère stapédienne persistante (ASP) gauche.

Discussion: Deux types d'anomalies artérielles peuvent être retrouvées dans le syndrome PHACE : la persistance d'artères embryonnaires et des anomalies des vaisseaux cérébraux. Une ASP peut ainsi être retrouvée dans le cadre d'un syndrome PHACE et il convient chez ces enfants de réaliser un suivi auditif régulier afin de ne pas méconnaitre un trouble auditif pouvant avoir des conséquences sur le développement de leur langage.

Mots Clés : artère stapédienne persistante, syndrome PHACE, hémangiomes, surdité unilatérale. 


\section{$\underline{\text { Introduction }}$}

Le syndrome PHACE décrit pour la première fois en 1996 par Frieden et al, est un syndrome rare découvert à la naissance [1]. Il associe des anomalies de la fosse postérieure $(\mathrm{P})$, des hémangiomes $(\mathrm{H})$, des anomalies artérielles (A), une coarctation de l'aorte (C) et des anomalies oculaires (E) [1].

Les anomalies artérielles sont de deux types: persistance d'artères embryonnaires et anomalies des vaisseaux cérébraux [2]. Aucun cas d'association de ce syndrome avec une artère stapédienne persistante (ASP) n'a été décrit auparavant dans la littérature.

Nous présentons le cas de la découverte d'une ASP dans le cadre d'un syndrome PHACE.

\section{$\underline{\text { Observation }}$}

Une enfant de 6 ans aux antécédents de syndrome PHACE découvert à sa naissance, a consulté pour une hypoacousie unilatérale gauche.

Son syndrome PHACE avait été découvert à la naissance devant un hémangiome infantile à forme tubéreux segmentaire des segments S1 (fronto-temporal), S2 (maxillaire) et S4 (frontonasal) gauche.

Une imagerie par résonance magnétique (IRM) encéphalique réalisée à la naissance avait permis de découvrir une hypoplasie de I' hémisphère cérébelleux gauche, sans atteinte controlatérale ni vermienne. L'artère vertébrale gauche naissait directement de la crosse aortique et le reste du polygone de Willis était sans anomalie. Il n'existait pas de cardiopathie ni d'anomalie oculaire. 
L'examen clinique ORL retrouvait des séquelles de l' hémangiome de la joue et du pavillon de l'oreille gauche (Figure 1) avec des tympans normaux.

L'audiométrie retrouvait une surdité de transmission de l'oreille gauche avec un Rinne de $10 \mathrm{~dB}$ en moyenne.

La tomodensitométrie (TDM) des rochers réalisée sans injection montrait un canal vasculaire naissant du canal carotidien gauche remontant le long du promontoire et passant entre les deux branches de l'étrier, au contact de sa branche antérieure, avant de sortir de l'oreille moyenne (Figure 2). On constatait l'absence du foramen spinosum gauche, confirmant la présence d'une ASP donnant naissance à l'artère méningée moyenne (AMM) gauche. Le foramen spinosum droit était visible donnant naissance normalement à l'AMM.

Devant la faible perte auditive de la patiente il a été décidé d'une surveillance clinique et audiométrique régulière.

\section{$\underline{\text { Discussion }}$}

Le syndrome PHACE est un syndrome rare cérébro-facial impliquant plusieurs segments consécutifs de la crête neural. La prédominance féminine a fait suspecter une anomalie dominante liée à l'X [3]. Le diagnostic est le plus souvent posé à la naissance devant des hémangiomes associés à des atteintes de la fosse postérieure (hypoplasie cérébelleuse, kyste arachnoïdien, dysgénésies corticales ou malformation de type Dandy-Walker) et des anomalies artérielles dans la grande majorité des cas unilatérales et homolatérales aux hémangiomes $[1,2]$.

Les anomalies artérielles sont de deux types :

- Les anomalies des vaisseaux cérébraux : dysplasies, sténoses, occlusions, hypoplasies voire agénésies des artères carotides, vertébrales et du tronc 
basilaire, origine ou trajet aberrant, persistance d'une artère trigéminale, anévrysmes sacculaire.

- La persistance d'artères embryonnaires telles que l'artère proatlantal segmentaire, l'artère ophtalmique primitive et l'artère hypoglosse primitive [2].

Une ASP est une anomalie vasculaire rare retrouvée chez environ $0.48 \%$ de la population [4, 5]. Elle correspond à la persistance d'une artère embryonnaire disparaissant normalement autour de la dixième semaine de gestation $[5,6]$.

Au cours du développement embryonnaire du système branchial, 6 paires d'arc aortiques se développant successivement sont formées reliant le sac aortique aux aortes dorsales paires. Le deuxième arc donne l'artère hyoïde $(\mathrm{AH})$ naissant de l'aorte dorsale (future artère carotide interne). L'artère stapédienne nait de l'AH à environ 4-5 semaines du développement embryonnaire. Elle se divise ensuite en une branche supérieure (supra-orbitaire) qui deviendra l'AMM et une branche inférieure (infra-orbitaire) qui deviendra l'artère infra-orbitaire et l'artère alvéolaire inférieure. Cette branche inférieure s'anastomosera avec l'artère pharyngée ventrale (future artère carotide externe) naissant du sac aortique. L'AH deviendra l'artère carotico-tympanique, branche de l'artère carotide interne. Avec l'involution de l'artère stapédienne, l'AMM née de l'artère carotide externe passe au travers du foramen spinosum. Lors de la persistance de l'artère stapédienne, l'AMM nait de cette dernière, donc du système carotidien interne, entrainant la disparition du foramen spinosum $[5,6]$.

La découverte d'une ASP est souvent faite de façon fortuite, devant une surdité de transmission ou des acouphènes pulsatiles. Elle serait à l'origine d'une ankylose stapédovestibulaire secondaire aux pulsations de l'artère sur les branches de l'étrier [5]. 
La réalisation d'une TDM permet de faire le diagnostic positif en recherchant une ASP associée à l'absence de foramen spinosum, une augmentation de calibre du canal du facial dans sa deuxième portion et parfois une carotide interne procidente $[4,5]$.

Il n'existait pas de procidence de l'artère carotide interne chez cette enfant sans doute en raison du fait que le syndrome PHACE est souvent associé à des sténoses progressives des artères intracérébrales secondaires à des proliférations concentriques de la paroi vasculaire réduisant progressivement leur lumière [2].

En cas d'ASP symptomatique avec surdité ou acouphènes pulsatiles, plusieurs auteurs ont décrit la réalisation d'une électrocoagulation ou d'une coagulation laser sans complication notamment sur le nerf facial ou sur l'audition $[4,5]$.

\section{Conclusion}

Le syndrome PHACE est une maladie rare dans laquelle la persistance d'artères embryonnaires dont l'ASP peut être retrouvée de façon unilatérale et homolatérale aux hémangiomes. Le diagnostic est posé le plus souvent sur une TDM des rochers. Il convient chez ces enfants de réaliser un suivi auditif régulier afin d'envisager, si la surdité venait à être handicapante et gênante pour le développement du langage une coagulation de cette artère.

Les auteurs déclarent ne pas avoir de conflits d'intérêts en relation avec cet article. 


\section{Références}

[1] Frieden IJ, Reese V, Cohen D. PHACE syndrome. The association of posterior fossa brain malformations, hemangiomas, arterial anomalies, coarctation of the aorta and cardiac defects, and eye abnormalities. Arch Dermatol 1996;132(3):307-11.

[2] Rotter A, Samorano LP, Rivitti-Machado MC, Oliveira ZNP, Gontijo B. PHACE syndrome: clinical manifestations, diagnostic criteria, and management. An Bras Dermatol 2018;93(3):405-411.

[3] Assari R, Ziaee V, Moghimi S, Akbari MR, Mirmohammadsadeghi A. PHACE(S) syndrome: Report of a case with new ocular and systemic manifestations. J Curr Ophthalmol 2017;29(2):136-138.

[4] Breheret R, Bizon A, Tanguy JY, Laccourreye L. [Persistent stapedial artery with otosclerosis]. Ann Otolaryngol Chir Cervicofac 2009;126(5-6):259-63.

[5] Hitier M, Zhang M, Labrousse M, Barbier C, Patron V, Moreau S. Persistent stapedial arteries in human: from phylogeny to surgical consequences. Surg Radiol Anat 2013;35(10):883-91.

[6] Silbergleit R, Quint DJ, Mehta BA, Patel SC, Metes JJ, Noujaim SE. The persistent stapedial artery. AJNR Am J Neuroradiol 2000;21(3):572-7. 
Figure 1: Séquelles de l'hémangiome hémifacial gauche avec atteinte de la joue et du pavillon de l'oreille gauche.

Figure 2: TDM des rochers de l'oreille gauche en coupe axiale montrant la persistance de l'artère stapédienne gauche. Flèche rouge : artère stapédienne persistante. 


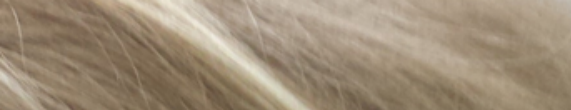

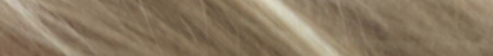

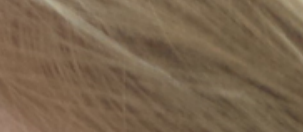
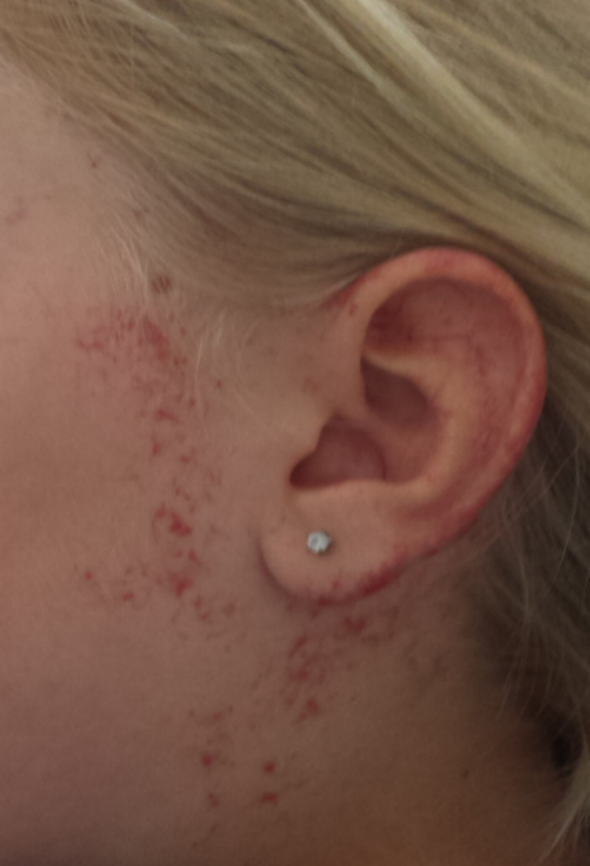
85. 32

ofyrexas

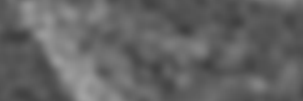

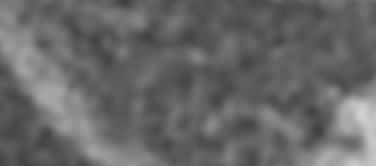

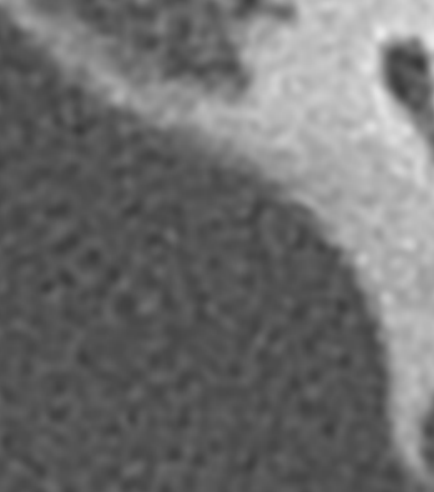

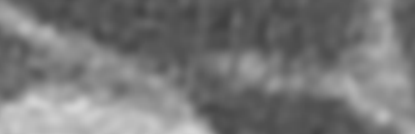

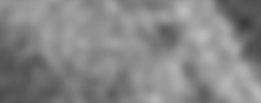

rcy

aryes on:

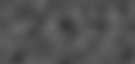

